\title{
Personalização das interações de um agente conversacional utilizando emoções e perfis de personalidade
}

\author{
Cleon Pereira Junior ${ }^{1}$, Adilmar Dantas ${ }^{2}$, Aline Abreu ${ }^{2}$, Matheus \\ Reis $^{2}$, Sara Melo ${ }^{3}$, Marcelo Nascimento ${ }^{2}$ Fabiano Dorça $^{2}$, Márcia Fernandes ${ }^{2}$ \\ ${ }^{1}$ IF Goiano - Campus Iporá - Iporá-GO - Brasil \\ ${ }^{2}$ Faculdade de Computação - UFU - Uberlândia-MG-Brasil \\ ${ }^{3}$ Faculdade de Engenharia Elétrica - UFU - Uberlândia-MG - Brasil \\ cleon.junior@ifgoiano.edu.br, akanehar@gmail.com, aline.lima@ufu.br, \\ matheuscunhareis30@gmail.com, sara.melodyahoo.com.br, \\ \{marcia, marcelo.nascimento, fabianodor\}@ufu.br
}

\begin{abstract}
Conversational Agents (CA), or chatbots, are applications able of generating dialogue, in text or speech form, with humans. This paper presents the development of an affective CA, based on personality profiles theory and emotions for personalized support to the student in the learning process. Thus, as an evaluation of the proposal, there was an integration of the approach in the Virtual Learning Environment Moodle. In addition, tests were carried out with real students, and the results obtained showed a potential in the interaction of personalized CAs to the particular and emotional characteristics of the student.
\end{abstract}

Resumo. Os Agentes Conversacionais (ACs), ou chatbots, são aplicações capazes de gerar diálogo, em forma de texto ou fala, com humanos. Este artigo apresenta o desenvolvimento de um AC afetivo, baseado na teoria perfis de personalidade e emoções para apoio personalizado ao estudante no processo de aprendizagem. Assim, como avaliação da proposta, houve uma integração da abordagem no Ambiente Virtual de Aprendizagem Moodle. Além disso, testes foram efetuados com estudantes reais e, os resultados obtidos apontaram uma potencialidade na interação de ACs personalizados às características particulares e emocionais do estudante.

\section{Introdução}

Os Ambientes Virtuais de Aprendizagem (AVAs) atualmente estão presentes como apoio ao ensino presencial, semipresencial e a distância. Esses ambientes permitem disponibilizar materiais de estudo, realizar avaliações contínuas e gerar feedbacks de forma automática e instantânea ao estudante [Arkorful and Abaidoo 2015]. Em alguns casos, também é possível encontrar fóruns e chats disponíveis, sendo que estes podem auxiliar na aprendizagem colaborativa.

O uso de AVAs não é um recurso recente, porém práticas de aprimoramento ainda são necessárias para a evolução do ensino. Um dos principais desafios atualmente é a personalização desses ambientes por meio de técnicas computacionais associadas às teorias pedagógicas. Para capacitar uma individualização no processo de aprendizagem, técnicas 
VIII Congresso Brasileiro de Informática na Educação (CBIE 2019)

Anais do XXX Simpósio Brasileiro de Informática na Educação (SBIE 2019)

de Inteligência Artificial (IA) podem ser utilizadas [Satu et al. 2015]. Para a personalização do ambiente, aspectos de personalidade e estados afetivos devem ser levados em consideração.

Uma das ferramenta que utilizam IA em sua construção e tem sido aplicada no contexto educacional são os Agentes Conversacionais (ACs). Um AC, comumente conhecido como chatbot, é capaz de gerar um diálogo em linguagem natural, na forma de texto ou fala com um ou mais humanos [Al-Zubaide and Issa 2011]. Estes agentes em um AVA apresentam vantagens se comparados com o processo de feedback somente pelo tutor, pois permite um suporte dinâmico ao estudante, podendo aumentar a motivação e melhores resultados [Kumar et al. 2007]. Com a existência de diversas soluções no mercado houve uma facilitação no desenvolvimento de chatbots e consequentemente a sua popularização. Na educação, por exemplo, são encontradas pesquisas que envolvem o uso de ACs para motivar trabalhos em pares [Kumar et al. 2007], auxiliar na mudança do estado afetivo do estudante [Ong and Soriano 2014], dentre outros.

Considerando a necessidade de personalização dos aspectos motivacionais aplicados aos estudantes em ambientes educacionais, esta pesquisa apresenta um chatbot afetivo aplicado em um AVA. O artigo está dividido em cinco seções. A seção a seguir apresenta os trabalhos correlatos envolvendo ACs, modelos afetivos para personalização e emoções. Em seguida, a seção 3 apresenta a abordagem geral, descrevendo cada módulo existente e as ferramentas para o desenvolvimento. A seção 4 apresenta os resultados obtidos com a aplicação da abordagem no Moodle. Por fim, a última seção traz as considerações finais apontando para possíveis trabalhos futuros.

\section{Fundamentação teórica e trabalhos correlatos}

As emoções interferem diretamente no processo de aprendizagem. Estudantes que se encontram em uma emoção prejudicial ao aprendizado não conseguem assimilar da melhor maneira possível o conteúdo que lhe é proposto. Nesta perspectiva, as pesquisas de [Izard 1984], afirmam que emoções negativas induzidas no estudante prejudicam o seu desempenho em tarefas cognitivas, assim como as emoções positivas possuem um efeito contrário [Jaques and Vicari 2005].

As seis emoções básicas (alegria, tristeza, raiva, medo, desgosto, surpresa) expressas pelos seres humanos são denominadas de emoções primárias. No âmbito acadêmico, essas emoções foram divididas em duas classes: emoções positivas ao aprendizado e emoções negativas ao aprendizado. Dentre essas seis emoções, apenas a emoção alegria é considerada como um estado afetivo ideal ao aprendizado. Sendo as outras cinco emoções, exceto em certas circunstâncias a emoção surpresa, são consideradas negativas a aprendizagem, responsáveis por inferir comportamentos pouco favoráveis no processo de ensino-aprendizagem [Chabot 2005].

O campo da personalidade refere-se às particularidades e diferenças do indivíduo. Na literatura, existem modelos que abordam a definição da personalidade e seus aspectos. Dentre eles, Big-Five Factors [John and Srivastava 1999], o modelo Myers-Briggs Type Indicator [Myers et al. 1985] e o modelo psicobiológico de Cloninger [Cloninger et al. 1993].

Baseado no modelo de [Cloninger et al. 1993], Chabot (2005) apresenta a teoria Perfis de Personalidade. Nesta teoria são exibidas três dimensões psicológicas, sendo 
VIII Congresso Brasileiro de Informática na Educação (CBIE 2019)

Anais do XXX Simpósio Brasileiro de Informática na Educação (SBIE 2019)

elas: i) A busca do Novo ii) Evitação da punição e dor; iii) Necessidade de Recompensa e Afeto. Em cada uma dessas dimensões são detalhadas características que definem o temperamento do indivíduo, com ênfase na aprendizagem. Em cada dimensão são atribuídos níveis e a combinação destes níveis resultam em nove perfis de personalidade, definidos por: temerário, teatral, excessivo, astucioso, meticuloso,afetivo, dócil, eremita e flexível.

Devida a variedade de informações, descritas por [Chabot 2005], que caracterizam os níveis alto e baixo em cada dimensão de personalidade, efetuar a classificação dos nove perfis apenas pelo comportamento observável do estudante torna-se uma tarefa não trivial. Sendo assim, o trabalho de [Melo et al. 2017] exibe uma classificação adequada dos perfis de personalidade, com uma precisão geral de 97,28\% obtidos por meio da técnica de Redes Neurais Artificiais. Além disso, foi determinado um conjunto ótimo de estratégias pedagógicas que podem ser utilizadas para interagir com estudantes utilizando estratégias manuais ou automáticas.

O desenvolvimento e aperfeiçoamento dos chatbots tiveram um crescimento considerável, porém ainda os classificam em fase de desenvolvimento [Rahman et al. 2017]. Existem plataformas específicas para facilitar o processo de desenvolvimento, na maioria dos casos essas soluções são responsáveis pelo processamento de linguagem natural dos bots, permitindo assim que os desenvolvedores se dediquem em outras personalizações, voltadas para o domínio do problema. Também é possível criar chatbots utilizando ontologias [Júnior et al. 2017], redes neurais profundas [Serban et al. 2017] ou simplesmente uma combinação de técnicas de IA com plataformas já existentes.

Com relação à afetividade, de forma mais estática e utilizando ontologias, é possível encontrar um chatbot integrado a um ambiente virtual de aprendizagem. Neste caso, o AC interage com crianças e recomenda conteúdos de acordo com o estado afetivo que estes aprendizes informam que estão. Com uma interface amigável, a cada exercício o estudante informa se está feliz ou triste. Além disso, uma ontologia de senso comum também é aplicada no modelo, com alguns filtros no conteúdo, pelo público-alvo se tratar de crianças [Ong and Soriano 2014].

Ainda relacionado ao uso de ACs afetivos, nota-se que estes são investigados desde a década de 90 [Walker et al. 1997]. Após quase três décadas, o assunto continua recente devido a evolução e aperfeiçoamento da capacidade de processamento computacional, o que permite que as técnicas, muitas vezes propostas sem personalização, sejam atualizadas de acordo com os interesses e personalidade do indivíduo que está interagindo com o agente.

\section{Abordagem Proposta}

Este trabalho apresenta o desenvolvimento e integração de um Agente Conversacional para fim educacional. No aspecto da personalização, este AC foi desenvolvido para responder estudantes de acordo com suas emoções e seu perfil de personalidade.

O primeiro passo da solução proposta requer a captura da personalidade do estudante. O trabalho em questão utilizou da teoria perfis de personalidade, pois a mesma apresenta, em seu desenvolvimento, uma ênfase na aprendizagem [Chabot 2005]. Como estratégia de coleta do perfil de personalidade, foi integrado ao ambiente virtual de aprendizagem um questionário composto por 62 questões, conforme apresenta [Melo et al. 2017]. 
VIII Congresso Brasileiro de Informática na Educação (CBIE 2019)

Anais do XXX Simpósio Brasileiro de Informática na Educação (SBIE 2019)

De acordo com [Chabot 2005] os perfis de personalidade devem estar associados com a emoção. Conforme apresentado por [Melo et al. 2017], existem diferentes regras que envolvem perfis de personalidade e emoção, portanto a recomendação deve ser personalizada levando em consideração os dois aspectos afetivos. Neste sentido, ao capturar o traço de personalidade, o próximo passo desta abordagem envolve o acompanhamento do estado emocional.

Neste trabalho inicialmente está sendo levado em consideração três emoções primárias (alegria, tristeza e raiva). Para a detecção dessas emoções, foi utilizada uma API (Application Programming Interface) desenvolvida pela Microsoft ${ }^{1}$. O módulo desenvolvido para detecção das emoções tem como função capturar o texto digitado pelo estudante, processá-lo e atualizar o estado emocional do estudante.

Para a avaliação geral da conversa entre o agente e o estudante é utilizada a técnica de regressão logística. Esta técnica estatística tem como objetivo produzir, a partir de um conjunto grande de observações, um modelo que permite a predição de valores tomados por uma variável categórica [Hosmer Jr et al. 2013]. Neste trabalho, a aplicação desta técnica estatística permitiu classificar a conversa como positiva ou negativa durante o decorrer de todo o diálogo de maneira rápida e eficiente.

Com relação a emoção, a captura por texto só foi possível de ocorrer por haver um agente estimulando o diálogo com o estudante. Para que o chatbot funcionasse corretamente foi observado a necessidade de implementar três diferentes ações para compor o módulo conversacional, sendo elas: afetiva; conhecimentos gerais; global. A Figura 1 representa o fluxo geral de funcionamento do $\mathrm{AC}$ para esta proposta.

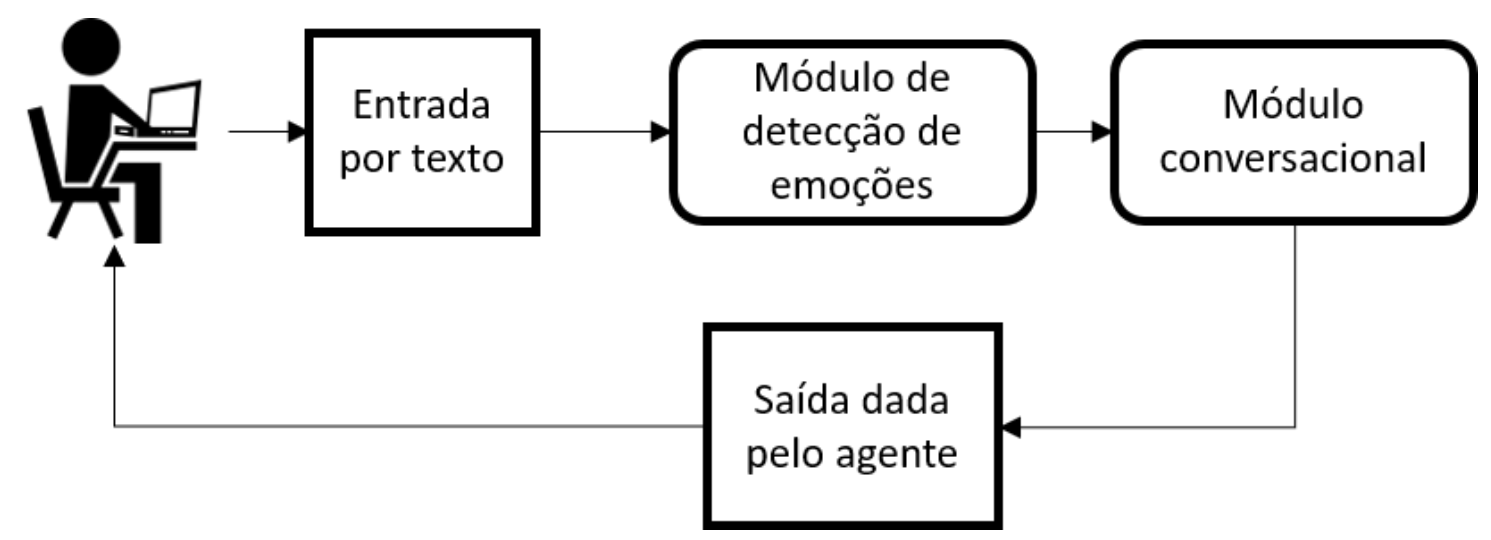

Figura 1. Fluxo geral de funcionamento do AC

O AC desenvolvido contou com o apoio do Dialogflow ${ }^{2}$ para classificar os textos e permitir uma resposta de acordo com a entrada do usuário. O Dialogflow é uma plataforma para construir interfaces de conversação para bots, aplicativos e dispositivos, usando processamento de linguagem natural e aprendizado de máquina. A construção da estrutura de conversação utilizada é feita a partir de intenções que representam um mapeamento entre o que usuário diz e qual ação deverá ser tomada pelo software e entidades. As entidades são ferramentas usadas para extrair valores de parâmetros de entrada de linguagem natural.

\footnotetext{
${ }^{1} \mathrm{https}: / /$ azure.microsoft.com/pt-br/services/cognitive-services/

${ }^{2}$ https://dialogflow.com/
} 
VIII Congresso Brasileiro de Informática na Educação (CBIE 2019)

Anais do XXX Simpósio Brasileiro de Informática na Educação (SBIE 2019)

Observando o fluxo representado na Figura 1, nota-se que o diálogo ocorre através da entrada de textos pelo usuário no momento de estudo por meio da plataforma de aprendizagem. A cada texto digitado, este é automaticamente classificado pela emoção (Microsoft Cognitive Services), conforme explicado anteriormente, e, em seguida, há a detecção da intenção (Dialogflow). Ao capturar uma intenção de acordo com a entrada de texto do usuário, a ferramenta foi programada para classificar qual ação de diálogo deve tomar com um determinado estudante. A seguir, uma breve explicação dos três módulos conversacionais:

- Diálogos genéricos: Ocorre quando o usuário questiona o agente sobre questões que não envolvem o estado afetivo. Ex: qual é o seu nome?; Quantos anos você tem?; Como você foi desenvolvido?.

- Diálogos afetivos: Neste caso, ocorre quando a mensagem enviada pelo usuário tem um cunho emocional e deve ser respondida de acordo com a personalidade. Ex: A tarefa está muito difícil; Estou muito feliz; Preciso de ajuda na atividade.

- Diálogos de conhecimento global: Desenvolvido para dar mais um suporte ao aprendiz, esta etapa consiste em tentar responder dúvidas do usuário através de conceitos definidos na wikipedia. Neste caso, é necessário que a intenção seja expressada em forma de curiosidade. Ex: O que é recursividade; $\mathrm{O}$ que é programação funcional; O que é chatbot.

Para responder aos diálogos afetivos e de conhecimento global, foi necessário o desenvolvimento de um Webhook. O Webhook é um web service que recebe e fornece informações em tempo real para outras aplicações. A finalidade do webhook, com relação aos diálogos afetivos, foi cadastrar respostas correlacionadas às intenções, juntamente com seus perfis de personalidade e emoções. Já em relação ao diálogo global, o webhook serviu para gerar uma conexão entre a aplicação e a wikipedia.
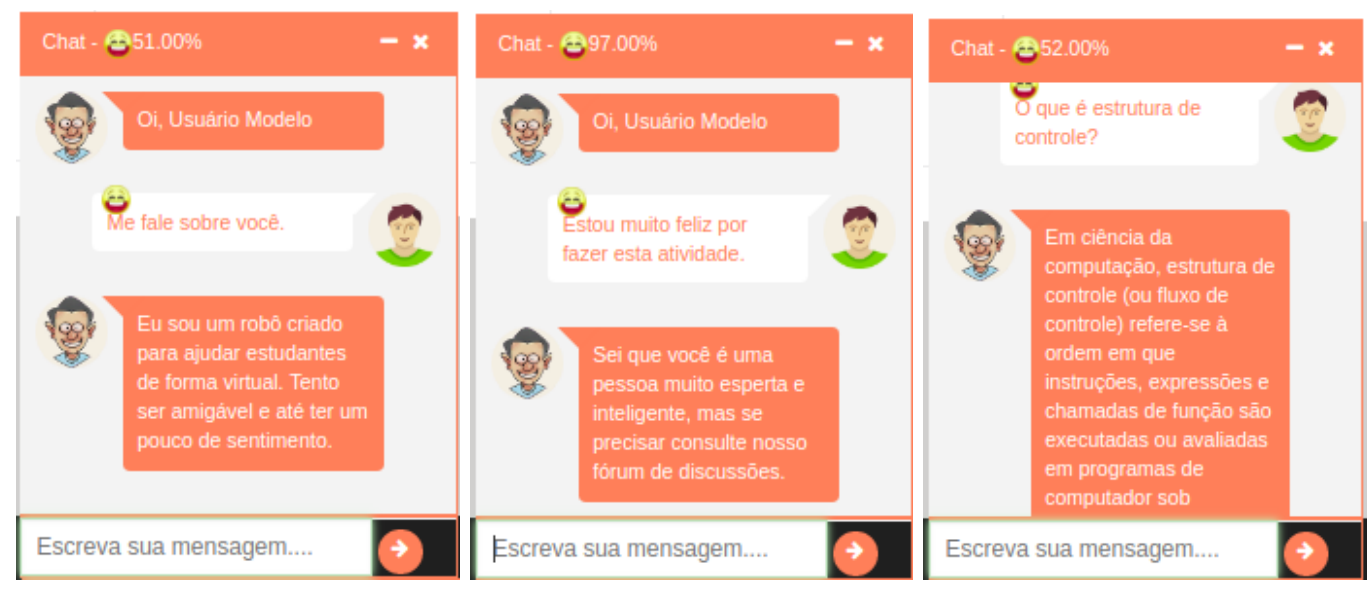

Figura 2. Exemplo do AC para os três níveis de diálogos. Chat 1 representa diálogo genérico, o chat 2 representa 0 diálogo afetivo e o chat 3 representa 0 diálogo global

A Figura 2 exemplifica o funcionamento dos três módulos conversacionais do agente. É possível notar que, independente do tipo de diálogo, a emoção é atualizada e o aprendiz consegue acompanhar em tempo real o que a aplicação tem retornado com relação à emoção. 
O AC desenvolvido tem capacidade de fornecer 2104 respostas distintas, sendo que 268 correspondem ao diálogo genérico e 1836 ao diálogo afetivo. Além disso, as respostas não são estáticas. Com o auxílio de técnicas de aprendizagem de máquina já presentes no Dialogflow, o bot é capaz de dar respostas distintas para uma mesma pergunta feita. Tal habilidade permite trazer a sensação de um diálogo entre dois seres humanos.

\section{Resultados}

Para avaliar o trabalho proposto, o módulo apresentado na seção anterior foi implementado no ambiente virtual de aprendizagem Moodle. Desta forma, o mesmo foi disponibilizado para uma turma de estudantes reais em uma disciplina de introdução à programação, com um total de 36 alunos frequentes. Esses alunos foram submetidos a realizar atividades no AVA (conforme já ocorria semanalmente na disciplina), neste caso com as adaptações necessárias para o funcionamento do chatbot.

No primeiro passo da avaliação foi realizado o preenchimento do questionário. Para isso foi criada uma atividade no AVA, explorando funcionalidades já existentes. Apesar da disponibilidade em forma de atividade, os estudantes foram avisados que era uma participação voluntária. O questionário foi preparado para que os dados oriundos deste fossem automaticamente tabulados de forma que pudesse ser interpretado pela rede neural proposta anteriormente para tal finalidade.

Um total de 16 estudantes responderam ao questionário disponibilizado, conforme apresentado pela Figura 4. Dos dezesseis estudantes que responderam ao questionário, foi possível detectar sete dos nove perfis de personalidade existentes na teoria proposta por Chabot [Chabot 2005]. Também foi possível observar uma presença maior do perfil de personalidade dócil. A partir destes dados, foram realizadas duas semanas de atividades.

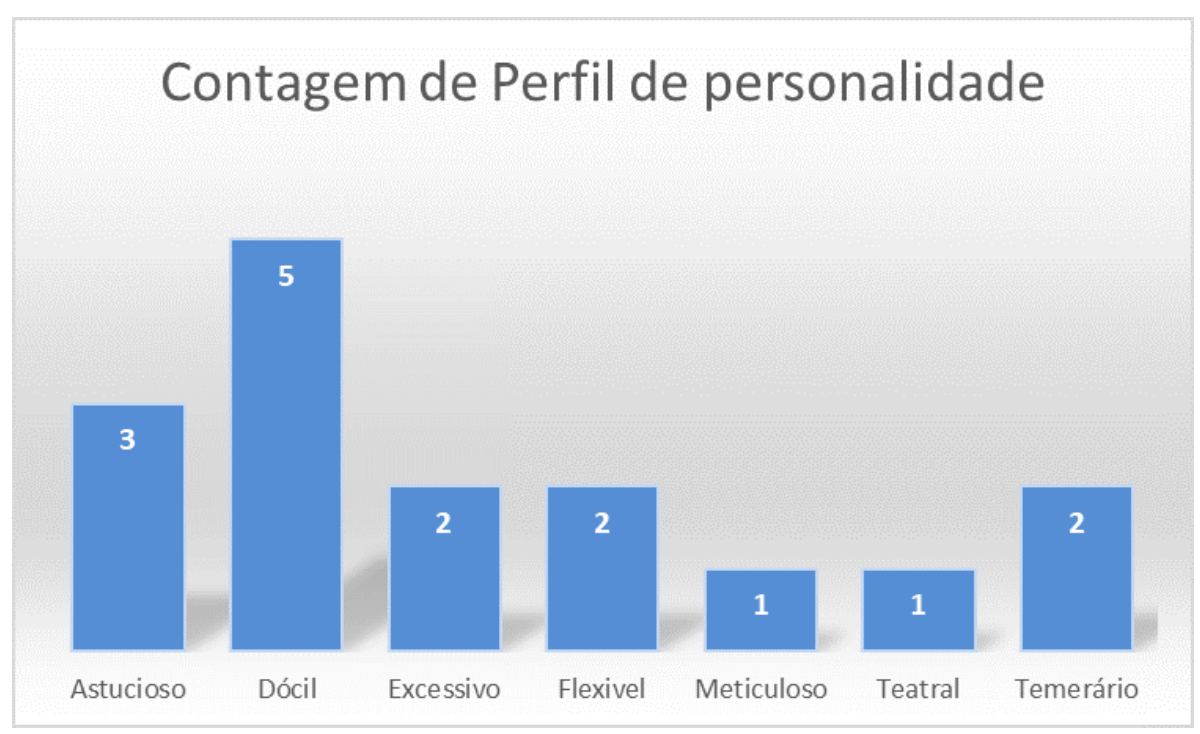

Figura 3. Gráfico dos perfis de personalidade classificados para os estudantes.

Com relação às duas semanas de atividades, estas foram propostas de forma regular para toda a turma. Assim como o chatbot também esteve disponível para todos. Tal prática foi necessária para não haver nenhuma diferenciação entre os alunos e também continuar o fluxo normal do curso. Apesar disso, somente os alunos que responderam o 
VIII Congresso Brasileiro de Informática na Educação (CBIE 2019)

Anais do XXX Simpósio Brasileiro de Informática na Educação (SBIE 2019)

questionário puderam ser analisados com relação ao diálogo afetivo por conta dos dados necessários.

A primeira semana houve um esforço em fazer com que ao menos um indivíduo de cada perfil executasse a atividade proposta utilizando o chatbot como apoio. Neste aspecto, cinco dos nove perfis utilizaram o AC. Todas as mensagens trocadas durante a atividade foram armazenadas em um banco de dados relacional para permitir uma análise mais detalhada da atuação do agente.

Fazendo uma análise mais detalhada dos alunos que utilizaram o chatbot como apoio durante a avaliação e escolhendo arbitrariamente um estudante de cada perfil apresentado, foi possível concluir que dois dos três módulos conversacionais (Afetivo e genérico) foram utilizados durante o diálogo com o chatbot. A Tabela 1 apresenta com mais detalhes os dados acerca dos diálogos obtidos para cada um dos perfis.

\section{Tabela 1. Dados quantitativos de troca de mensagens obtidos na primeira se-} mana.

\begin{tabular}{l|l|l|l|l} 
Perfil & Mensagens Enviadas & afetivo & Genérico & Global \\
\hline Flexivel & 14 & 3 & 11 & - \\
\hline Astucioso & 25 & 1 & 24 & - \\
\hline Temerário & 26 & 12 & 14 & - \\
\hline Dócil & 3 & 1 & 2 & - \\
\hline Excessivo & 15 & 9 & 6 & -
\end{tabular}

A Tabela 2 apresenta os dados quantitativos durante toda a avaliação da ferramenta. No contexto geral, somando as duas semanas de experimento, dezenove estudantes utilizaram o chatbot. Destes dezenove, nove deles não preencheram o questionário, portanto não foi possível detectar o perfil de personalidade, porém o AC ainda era possível de ser utilizado, neste caso não personalizado.

Dos dados analisados, foi observado também uma variação nas emoções dos estudantes durante o diálogo, sendo que dos cinco perfis, somente o astucioso se manteve em todo o momento na alegria. Além disso, somente um dos estudantes do perfil dócil encerrou o diálogo com uma frase que demonstrava raiva, os demais finalizaram com a média global e a última frase com o estado atual de alegria. Desta forma, o uso do AC demonstrou uma forma positiva com relação ao estado do estudante durante a execução de atividades em um AVA.

Tabela 2. Dados quantitativos de troca de mensagens entre os alunos e o chatbot.

\begin{tabular}{l|l|l|l|l|l} 
Perfil & Número de usuários & Mensagens Enviadas & Afetivo & Genérico & Global \\
\hline Teatral & 1 & 7 & - & 7 & - \\
\hline Flexível & 2 & 18 & 3 & 15 & - \\
\hline Excessivo & 1 & 43 & 9 & 34 & - \\
\hline Astucioso & 1 & 30 & 1 & 29 & - \\
\hline Dócil & 3 & 45 & 4 & 37 & 4 \\
\hline Temerário & 1 & 29 & 12 & 17 & - \\
\hline Sem perfil & 9 & 160 & 7 & 109 & 44
\end{tabular}


VIII Congresso Brasileiro de Informática na Educação (CBIE 2019)

Anais do XXX Simpósio Brasileiro de Informática na Educação (SBIE 2019)

Com relação à segunda semana de testes, dezenove dos trinta e seis estudantes entregaram as atividades em tempo hábil. Dos alunos que realizaram a tarefa, 73,68\% (catorze estudantes) utilizaram o AC como apoio para a realização. Este resultado demonstra uma motivação no uso de ACs afetivos personalizados no contexto de aprendizagem.

\section{Considerações Finais}

Este trabalho apresentou uma abordagem utilizando um AC afetivo como suporte dinâmico durante o desenvolvimento de atividades em um AVA. Os testes iniciais demonstraram uma potencialidade de um AC personalizado para suporte online e cria expectativas para aprimoramento e diferentes investigações com este tipo de ferramenta.

Os resultados apresentados são iniciais e aplicações em turmas distintas podem auxiliar na coleta e análise dos dados. Além disso, verificar quais perfis apresentam mais sucesso / fracasso com uso de ACs podem aprimorar a pesquisa em computação afetiva e verificar diferentes tipos de suporte dinâmico para perfis distintos.

Como trabalhos futuros pretende-se criar mais uma ação do diálogo conversacional que deve dar resposta acerca do tempo de atividade, prazos de entrega, notas, dentre outras informações que são possíveis de capturar a partir das tabelas do Moodle, operando como um assistente virtual do estudante. Além disso, espera-se que com o uso de técnicas de análise conversacional seja possível a formação de grupos de acordo com o diálogo apresentado individualmente entre o aluno e o AC.

\section{Agradecimentos}

Os autores agradecem ao Instituto Federal de Educação, Ciência e Tecnologia Goiano (IF Goiano) e à Universidade Federal de Uberlândia (UFU), pelo apoio à esta pesquisa.

\section{Referências}

Al-Zubaide, H. and Issa, A. A. (2011). Ontbot: Ontology based chatbot. In Innovation in Information \& Communication Technology (ISIICT), 2011 Fourth International Symposium on, pages 7-12. IEEE.

Arkorful, V. and Abaidoo, N. (2015). The role of e-learning, advantages and disadvantages of its adoption in higher education. International Journal of Instructional Technology and Distance Learning, 12(1):29-42.

Chabot, D. (2005). Pedagogia emocional-sentir para aprender. Sa Editora.

Cloninger, C. R., Svrakic, D. M., and Przybeck, T. R. (1993). A psychobiological model of temperament and character. Archives of general psychiatry, 50(12):975-990.

Hosmer Jr, D. W., Lemeshow, S., and Sturdivant, R. X. (2013). Applied logistic regression, volume 398. John Wiley \& Sons.

Izard, C. E. (1984). Emotion-cognition relationships and human development. Emotions, cognition, and behavior, pages 17-37.

Jaques, P. and Vicari, R. (2005). Pat: um agente pedagógico animado para interagir efetivamente com o aluno. RENOTE, 3(1). 
VIII Congresso Brasileiro de Informática na Educação (CBIE 2019)

Anais do XXX Simpósio Brasileiro de Informática na Educação (SBIE 2019)

John, O. P. and Srivastava, S. (1999). The big five trait taxonomy: History, measurement, and theoretical perspectives. Handbook of personality: Theory and research, 2(1999):102-138.

Júnior, C. P., Francisco, R., Silva, L., Veiga, E., Fernandes, M., and Dorça, F. (2017). Uso de ontologias para agentes conversacionais no contexto de ensino-aprendizagem: Uma revisão sistemática da literatura. In Brazilian Symposium on Computers in Education (Simpósio Brasileiro de Informática na Educação-SBIE), volume 28, page 183.

Kumar, R., Rosé, C. P., Wang, Y.-C., Joshi, M., and Robinson, A. (2007). Tutorial dialogue as adaptive collaborative learning support. Frontiers in artificial intelligence and applications, 158:383.

Melo, S. L., Dantas, A. C., and Fernandes, M. (2017). Modelo do estudante baseado em emoções e perfis de personalidade para recomendação de estratégias pedagógicas personalizadas. In Brazilian Symposium on Computers in Education (Simpósio Brasileiro de Informática na Educação-SBIE), volume 28, page 967.

Myers, I. B., McCaulley, M. H., and Most, R. (1985). Manual, a guide to the development and use of the Myers-Briggs type indicator. Consulting Psychologists Press.

Ong, E. C. J. and Soriano, Z. C. (2014). A conversational agent to shift students' affect state. In International Workshop on Empathic Computing, pages 86-97. Springer.

Rahman, A., Al Mamun, A., and Islam, A. (2017). Programming challenges of chatbot: Current and future prospective. In Humanitarian Technology Conference (R10-HTC), 2017 IEEE Region 10, pages 75-78. IEEE.

Satu, M. S., Parvez, M. H., et al. (2015). Review of integrated applications with aiml based chatbot. In 2015 International Conference on Computer and Information Engineering (ICCIE), pages 87-90. IEEE.

Serban, I. V., Sankar, C., Germain, M., Zhang, S., Lin, Z., Subramanian, S., Kim, T., Pieper, M., Chandar, S., Ke, N. R., et al. (2017). A deep reinforcement learning chatbot. arXiv preprint arXiv:1709.02349.

Walker, M. A., Cahn, J. E., and Whittaker, S. J. (1997). Improvising linguistic style: Social and affective bases for agent personality. In Proceedings of the First International Conference on Autonomous Agents, AGENTS '97, pages 96-105, New York, NY, USA. ACM. 